\title{
E-Health Readiness Assessment for E-Health Framework for Africa: A Case Study of Hospitals in South Africa
}

\author{
Alfred Coleman ${ }^{1}$, Marlien E Herselman ${ }^{2}$ and Dalenca Potass ${ }^{3}$ \\ ${ }^{1}$ Tshwane University of Technology, Soshanguve Campus, South Africa \\ ${ }^{2}$ Living Labs, Meraka Institute, CSIR, South Africa \\ ${ }^{3}$ School of Information and Communication Technology, Nelson Mandela Metropolitan \\ University, South Africa \\ colemana@tut.ac.za, mherselmanecsir.co.za, \\ Dalenca.Pottas@nmmu.ac.za
}

\begin{abstract}
This study assessed e-healthcare readiness of rural and urban hospitals in North West Province of South Africa. Outcome of assessment led to creation of e-health architectural framework for e-health solutions. Assessment was conducted in usage of ICT in patient healthcare record system, processes and procedures in consultation among healthcare professionals, prescription of medication, referral of patients and training of healthcare professionals in ICT usage. The study was in two phases and six hospitals were selected. E-healthcare readiness assessment focusing on need, technological, engagement and social acceptance readiness were assessed. Data collected used group interviews and qualitative questionnaires. Findings showed that computers were not used for clinical duties and no e-health solutions were found. E-health Maturity Level was at level zero. Recommendations and compilation of Provincial E-Health Framework (PEHF) were made. The findings were unexpected and therefore, of great benefit to healthcare institutions which intend to implement e-health initiatives in hospitals.
\end{abstract}

Keywords: Technology readiness assessment, e-health, patient electronic health records, electronic prescription.

\section{Introduction}

HIV pandemic in most African countries has not only caused considerable strain on various national healthcare systems, but has increased the number of orphans, reduced productive human capital and productivity, eroded knowledge and skills, put pressure on national budgets, increased the poverty-stricken populace and reduced the quality of life, health and wellness[1]. E-health as one of the supportive systems within the healthcare system has great potential to address the challenges facing healthcare systems in developing countries. The recognition of Information and Communication Technology (ICT) in healthcare is not an end unto itself but a means to an end. The 
successful introduction of ICT in healthcare requires the examination of complex political, organisational and infrastructural factors, including a readiness factor [2].

The White Paper on transformation of the public healthcare system in South Africa (SA) reveals that majority of the population has inadequate access to basic healthcare services and that the greater percentage of this population lives in rural communities [3].The White Paper also indicates that majority of South Africans receive their medical care from government-run clinics and hospitals [4]. The North West Province Health Department (NWPHD) has invested substantial sums of money in the district and provincial health services, health facilities management and healthcare support services, including ICT, in an effort to improve the work processes of healthcare professionals in order to promote efficient delivery of healthcare services. Despite the high investment in the healthcare system, many healthcare professionals in NWP do not receive the benefits for which ICT can provide to improve on their work processes.

This article reports on how ICT can be used to promote efficiency in the work processes within the hospitals to deliver quality healthcare services to the people in North West Province (NWP). To operationalise this objective, an e-health readiness assessment of rural and urban hospitals was conducted. Outcome of the assessment led to compilation of e-health framework to improve healthcare professionals' work processes. The assessment investigated effective and efficient use of ICT in patient record systems, processes in consultation, prescription, referrals and training.

This research has contributed to the body of knowledge in e-health in two ways. Firstly, a set of recommendations has been drawn from the research data to help bridge the gap between current levels of ICT usage in healthcare to attain a higher level where there is integration of e-health adoption in the hospitals. Secondly, a PEHF for the hospitals in NWP has been compiled, based on the data drawn from the research.

\subsection{E-health and E-healthcare Readiness Assessment Theories}

The World Health Organization [5] defines e-health as "being the leveraging of ICT to connect providers, patients and governments; to educate and inform healthcare professionals, managers and consumers; to stimulate innovation in healthcare delivery and health system management and to improve our healthcare system'. Eysenbach [6] refers to e-health as 'a concerted effort undertaken by leaders in healthcare and hitech industries to fully harness the benefits available through convergence of the Internet and healthcare'. The advent of e-health seems fitting to address both opportunities and challenges in the healthcare sector but the question is, are hospitals in NWP ready to embrace the full potential of e-health?

Currently there are six assessment models which are commonly used to assess ehealth readiness in the health environment. These models [3], [8], [7], provide different dimensions which can be utilized in assessing e-healthcare readiness. These are core readiness, engagement readiness, structural readiness and non-readiness. Another component, Technology Acceptance Model [9], is included for use in developing countries, especially in rural areas. The principal components adopted for this e-health readiness assessment included technology acceptance construct which is vital to the introduction of any technology in rural hospitals. 


\section{Methods}

Qualitative research design employing a multiple case study approach was used. Data from selected urban and rural hospitals were collected, using group interview and qualitative questionnaire. The questionnaire instrument was used in addition to the group interview because it promoted reliability and validity of the data. The data were analyzed by utilizing [10] case study analysis template and [11] guidelines for case study analysis. The group interview and questionnaire items were formulated in accordance with the categories of background/history of hospitals, hospital infrastructure, ICT access level, including ICT availability, accessibility and usability; and e-health solutions including availability, accessibility and usability.

There are five regions in the province and all the regions were taken into account by selecting a hospital from each region. The selected hospitals were Rustenburg, Taung, Ganyesa, Klerksdorp, Christian and Reivilo Hospital. Rustenburg and Klerksdorp Hospitals are urban hospitals while Taung, Christiana and Reivilo Hospitals are rural hospitals.

\subsection{Participants}

A purposive sampling was applied to select respondents from the hospitals in order to achieve the goals of the study. A total of 48 respondents were selected. The selected respondents from each hospital had the following categories: 2 administrators, 2 general doctors, 2 professional nurses and 2 assistant nurses. The respondents were informed prior to the group interviews and the completion of the questionnaires that participation in the research was voluntary and that any information provided would be treated as confidential.

\subsection{Research Instruments}

\section{Group Interview}

A group interview was conducted in each hospital. The interviewees were of diverse age, ethnicity, gender and educational levels. The purpose of the interview was to determine how the healthcare professionals perceive the usefulness and potential benefits of e-patient record, e-prescription, e-consultation, e-referrals and e-training systems. The interview questions are attached to the full research report.

\section{Questionnaire}

The questionnaire instrument consisted of two sets of questions. The first set of questions was administered to hospital administrators whilst the second was for general doctors, nurses and assistant nurses. The purpose of the first set of questions was to establish the background history, the settings of the hospitals and the existing ICT infrastructure in these hospitals. The second set of questions was to establish baseline data for processes and procedures in keeping patient health records, consultation among healthcare professionals, prescription and referral processes. The questions were drawn from e-health readiness assessment framework [9]. 


\section{Results}

This section presents the findings and proposes some recommendations which served as guiding principles for the development of e-health solution.

\subsection{Questionnaire Results}

\section{Availability of Healthcare Professionals in Rural and Urban Hospitals}

The findings indicate that there is a widespread shortage of doctors in rural hospitals in NWP. An average ratio of 1 doctor to 18000 patients for rural hospitals as compared to1 doctor to 9000 patients in urban hospitals was found. These hospitals are all stateowned healthcare institutions.

\section{ICT Availability and Accessibility in Rural and Urban Hospitals}

The findings reveal that urban hospitals have more ICT equipment than rural hospitals. Internet connection is more reliable in terms of connectivity and speed in urban hospitals. In rural hospitals, connectivity and speed of Internet services are often affected by poor telephone lines and interruption of electricity power supply. The average ratio of number of computers to doctors in rural hospitals is 1:3 while it is 1:2 in urban hospitals. However, data obtained from the e-health readiness assessment revealed that there were no computers in the doctors' consultation rooms. It was ascertained that doctors do not use computers for their clinical duties. Again nurses and assistant nurses do not use computers for their clinical work. These computers are used by hospital administrative staff for capturing patients' demographic information and revenue collection. This means that even though there are computers in the hospitals, they are used for activities which are not directly related to clinical work like e-patient health record keeping.

Healthcare professionals' use of computer was also assessed. Healthcare professionals in urban hospitals use computers/Internet daily for searching for information but not for carrying out their clinical duties. Healthcare professionals in rural hospitals use computers weekly or monthly.

\subsection{Group Interview Results}

E-health Solution, Availability and Accessibility in Rural and Urban Hospitals, and Perceived Usefulness in Rural and Urban Hospitals

In comparing urban and rural hospitals in terms of e-health solution availability and accessibility, the following findings emerged: There is no e-patient health record system, e-consultation, e-prescription, e-referral and e-training systems in both urban and rural hospitals in NWP. Participants indicated that installation of e-patient health record system will save time when recording patient information, diagnosing and in the treatment of patients. One participant stated "Patients who do not get their records after a long time, the e-patient record system will help to safeguard this". It became evident that e-patient record system will improve communication and eliminate 
patients who collect medication from multiple hospitals with duplicate paper prescription. One respondent stated, "It will eliminate ghost patients and before a patient arrives at the pharmacy, the pharmacist will have the information about the patient".

It emerged that referrals of patients from a lower level hospital to a higher level hospital were done through the use of paper notes. Patients are given referral letters (except in emergency cases) which they take to the referred hospital. Patients often misplace these letters and end up not going to the referred hospital. One participant indicated, "E -referral will help because you refer patients but on the way they get lost or don't go. If it is done electronically, the referred hospital will know that such a time we expect this patient".

There is Internet facility in both urban and rural hospitals but the Internet is limited to searching information and sending e-mails. Both urban and rural hospitals have PAAB system which is used to collect and send patient demographic information to NWPHD's head office monthly. Urban hospitals have tele-radiography facility which is used for sending X-ray images between Klerksdorp and Rustenburg Hospitals.

\section{Discussion}

\subsection{Availability of Healthcare Professionals in Rural and Urban Hospitals}

An average ratio of 1 doctor to 18000 patients for rural hospitals as compared to 1 doctor to 9000 patients in urban hospitals was noted. Since these are state-owned healthcare institutions, the ratio of doctors to number of patients, both in rural and urban hospitals, is higher than that quoted by the [12] report. The report [12] focused on both private and state-owned hospitals in SA, while this research investigated only state-owned hospitals in NWP in SA. The shortage of doctors in rural hospitals has to drive investment into e-health technologies like e-consultation, to enable non-doctors healthcare professionals such as nurses and midwifes to perform more advanced functions through synchronous consultation with professional doctors.

\subsection{ICT Availability and Accessibility in Rural and Urban Hospitals}

It was found that more ICT equipment is needed in both rural and urban hospitals if ehealth solutions are to be implemented. Internet connection also needs upgrading. ICT infrastructure in both urban and rural hospitals is not integrated to be able to work together within and across urban and rural hospitals. This confirms research conducted in the Eastern Cape Province of SA [13].

\subsection{E-health Solution, Availability and Accessibility in Rural and Urban Hospitals, and Perceived Usefulness in Rural and Urban Hospitals}

The findings showed that the Internet facility in both rural and urban hospitals is limited to searching information and sending e-mails. Urban hospitals have teleradiography facility which enables them to send $\mathrm{x}$-ray images. These findings place both the urban and rural hospitals at Level 0 (The Baseline) on the E-health Maturity Curve as illustrated in Figure 1 below. 
Source: [14]

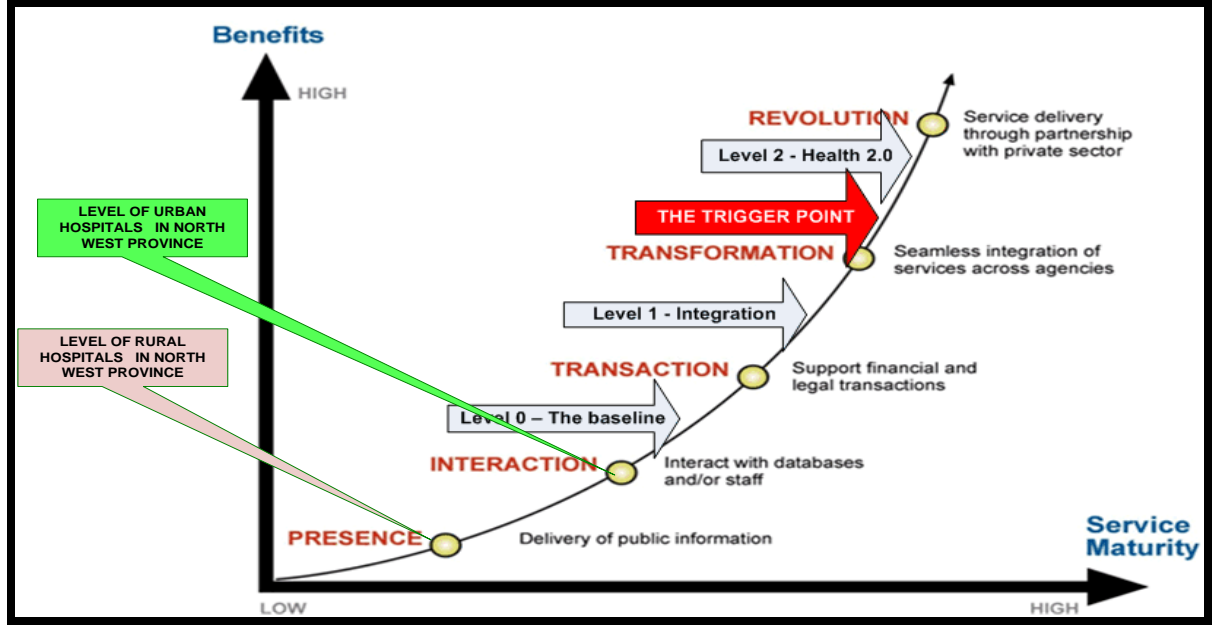

Fig 1: E-health Maturity Curve

Figure 1 illustrates that the level of e-health application in rural hospitals is at the Presence stage which is classified as Level 0 (The baseline). At this stage there is a non-interactive website where the main intent is to disseminate information. Thus, rural hospitals are able to receive and send information from and to NWPHD through e-mails and the PAAB system.

The level of e-health application in urban hospitals is at the Interaction stage which is also at Level 0 (The baseline). However, the Interaction stage offers services that are more advanced than the Presence stage. Thus, urban hospitals are able to send and receive x-ray images through the tele-radiography facility. Despite the availability of these ICT infrastructure facilities, the ICT systems are not integrated to be able to work together across departmental and organizational boundaries to use information that has been provided by the patient.

\section{The need for Provincial E-health Framework (PEHF)}

A major setback which became evident is that despite the availability of the ICT facilities and other ICT infrastructure in both urban and rural hospitals, the ICT systems are not integrated to work together within and across the hospitals to allow healthcare professionals to gain the benefits of e-health solutions and applications. These findings place both rural and urban hospitals at Level 0 on the E-Health Maturity Curve (cf Fig1). Therefore, it is imperative that a special e-health framework be compiled based on these findings to move the e-health application usage in these hospitals from Level 0 to Level 2 (Healthcare 2.0) on the E-Health Maturity Curve by ensuring that Level 1(Integration) is effectively and efficiently achieved. Therefore, the PEHF was compiled (cf Fig 2). 


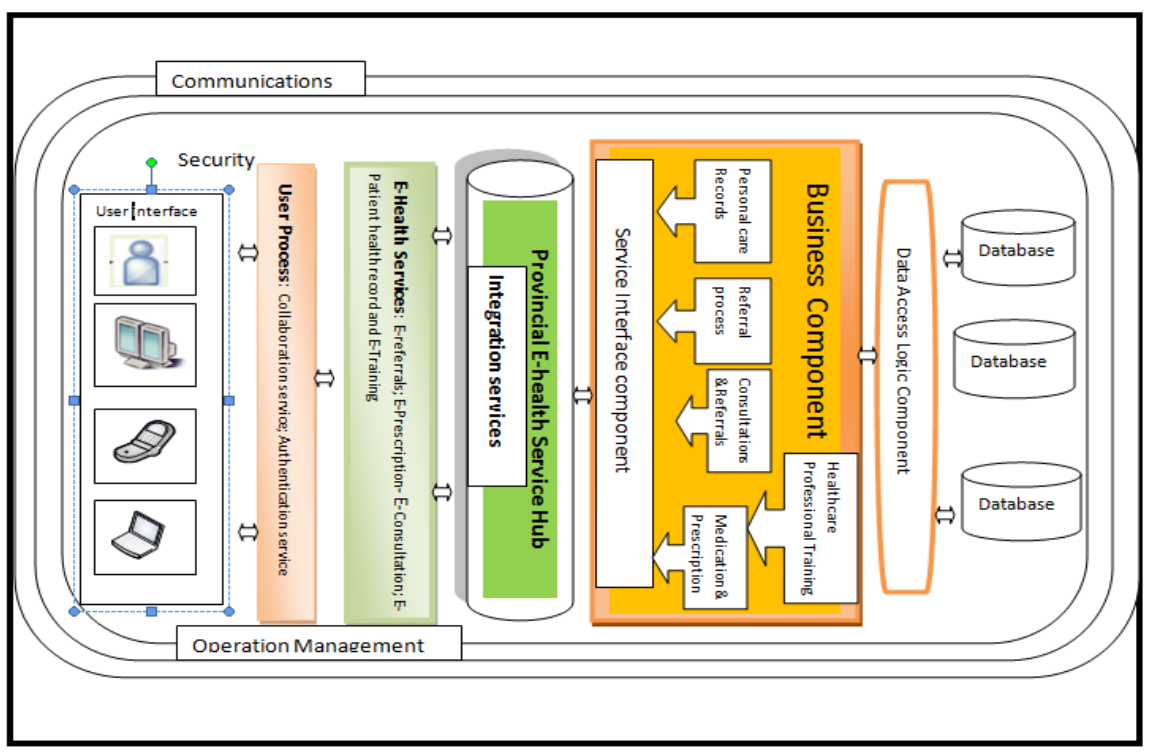

Fig 2: PEHF Architecture

The PEHF architecture emphasizes the integration of services within local hospitals and avails these services to the web through a provincial e-health services hub. This component incorporates the business services of the hospitals and transforms them from physical and paper phase to the electronic phase through the service hub. This represents the migration from the physical normal business and paper services of the hospitals to web-based services.

\section{Conclusion}

Having reviewed the literature on e-health and e-healthcare assessment, conducted the e-healthcare assessment in the selected hospitals, and considering the findings derived from the hospitals' assessment, this article provides the following conclusion:

-ICT infrastructure in both urban and rural hospitals is not integrated to work together within and across hospitals to allow healthcare professionals to gain the benefits of ehealth solutions and applications

-E-health applications in the work processes in the hospitals are at the Presence and Interaction stages on E-Health Maturity Curve which is Level 0 (The Baseline).

Based on the above conclusion, the following recommendations are made:

-ICT systems within each hospital and across hospitals in NWP need to be integrated in order to facilitate e-consultation by using an integrated network which will assist healthcare professionals to consult with peers and specialists for professional advice and information; and

-Inter-operability of systems to bring together the diverse systems and data sources into a coherent, controlled environment, create a network of healthcare delivery and 
incorporate existing e-health applications like PAAB and tele-radiography into one ehealth framework.

In conclusion, this article has provided the PEHF which will move the work processes of healthcare professionals from Level 0 to Level 2 (Healthcare 2.0) on the E-health Maturity Curve, leading to efficient and effective delivery of healthcare services to the people in NWP.

\section{Reference}

1. Botswana Government: Status of the National Response to the UNGASS Declaration of Commitment on HIV/AIDS. Gaborone: National Aids Coordinating Agency (2003)

2. Jennett, P., Jackson, A., Healy, T. et al.: A Study of a Rural Community's Readiness for Telehealth. J Telecare. 9, 259--63 (2003)

3. Department of Health (DoH). Documents and Reports.: North West Department of Health, http://www.nwhealth.gov.za/nwhealth_web/doc_reports.php

4. Rasmeni, M.: Budget Speech for the Department of Health for the Financial Year 2007/2008. Presented by the MEC for Health, at the North West Provincial Legislature. http://www.nwhealth.gov.za/nwhealth_web/dir_prog.php

5. World Health Organization (WHO).: Developing Health Management Information Systems: A Practical Guide for Developing Countries, Geneva (2004)

6. Eysenbach, G.: What is e-Health? J. Med Internet Research. 3 (2), 20 (2001)

7. Campbell, J.D., Harris, K.D., Hodge, R.: Introducing Telemedicine Technology to Rural Physicians and Settings. J. Fam Pract. 50, 419--24. Canada (2001)

8. Demiris, G., Oliver, D.R.P., Porock, D., Courtney, K.: Home Telehealth: The Missouri Telehospice Project: Background and Next Steps. Home Health Care Technology Report, 1(49), 55--7 (2004)

9. Ojo, S.O., Olugbara, O.O., Emuoyibofarhe, O.J.: Formal Model for e-Healthcare Readiness Assessment in Developing Country Context. In: Innovations in Information Technology. IIT '07. 4th International Conference (2007)

10. Creswell, J. W.: Qualitative Inquiry and Research Design. Choosing among Five Approaches, 2nd ed. Sage Publications, London (2007)

11. Klein, H.K., Myers, M.D.: A Set of Principles for Conducting and Evaluating Interpretive Field Studies in Information Systems. Management Information Systems Quarterly, 23(1), 67--94 (1999)

12. United Nations Framework Convention on Climate Change (UNFCCC).: Development and Transfer of Technologies. Workshop on Enabling Environment for Technology Transfer. www.unfccc.int/resource/docs

13. Nkqubela, L.R., Herselman, M.E.: ICT Application as E-health Solutions in rural Healthcare in the Eastern Cape of South Africa. Health Information Management J.: 39(1). (2010)

14. Jarvis, B., Fortunov, I., Bachour, T.: Connect Health Framework Architecture and Design Blue Print. www.microsoft.com/.../articles 